\title{
Germanica
}

\section{La rupture temporelle de 1989 et sa représentation dans la littérature, le cinéma et le théâtre}

\section{Carola Hähnel-Mesnard}

\section{OpenEdition}

\section{Journals}

Édition électronique

URL : https://journals.openedition.org/germanica/10524

DOI : 10.4000/germanica.10524

ISSN : 2107-0784

Éditeur

Université de Lille

Édition imprimée

Date de publication : 28 juin 2021

Pagination : 7-16

ISBN : 978-2-913857-47-6

ISSN : 0984-2632

\section{Référence électronique}

Carola Hähnel-Mesnard, « La rupture temporelle de 1989 et sa représentation dans la littérature, le cinéma et le théâtre », Germanica [En ligne], 68 | 2e trimestre 2021, mis en ligne le 28 juin 2021 consulté le 03 janvier 2023. URL : http://journals.openedition.org/germanica/10524 ; DOI : https:// doi.org/10.4000/germanica.10524 


\section{LA RUPTURE TEMPORELLE DE 1989 ET SA REPRÉSENTATION DANS LA LITTÉRATURE, LE CINÉMA ET LE THÉÂTRE}

Carola Hähnel-Mesnard

Université de Lille

\section{Une autre perception du temps}

Lorsque le mur de Berlin tombe le 9 novembre 1989, annonçant la fin de la RDA et de ses particularités politiques, économiques et socioculturelles, la perception que les Allemands de l'Est avaient du temps se trouve brusquement modifiée. Presque simultanément à l'événement, la littérature enregistre ce changement temporel. Ainsi, dans son cycle de poèmes «Sept télégrammes » rédigé à cette occasion, Durs Grünbein note à la date du «12/11/89": « Reviens à toi, poème. Maintenant, le mur de Berlin est tombé. / Chagrin de l'attente, ennui au pays étroit de Hegel / Passé comme un silence d'acier... [...]. » Et : « Lentement, les montres s'affolent, chacune à son rythme. $\gg^{1}$ Le poème de Grünbein révèle le passage d'une perception du temps propre à la RDA où dominent « l'attente » et «l'ennui » vers une nouvelle sensation du temps perceptible dès la chute du Mur : les horloges, manifestement à l'arrêt jusque-là, se remettent lentement en marche, mais à des rythmes différents - comme si après une période collective d'arrêt, le temps pouvait à nouveau être vécu différemment et, surtout, individuellement. D'autres auteurs expriment plus

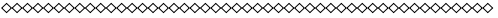

1. - Durs Grünbein, «12/11/89» (traduit de l'allemand par Silke Schauder), in : Après l'Est et l'Ouest. Volker Braun, Oskar Pastior, Bert Papenfuß, Durs Grünbein, Paris, Textuel, 2001, p. 168. Pour l'original : « Komm zu dir Gedicht, Berlins Mauer ist offen jetzt./Wehleid des Wartens, Langeweile in Hegels Schmalland/Vorbei wie das stählerne Schweigen... [...]/ [...] /Langsam kommen die Uhren auf Touren, jede geht anders » (D. Grünbein, Schädelbasislektion, Frankfurt a. M., Suhrkamp, 1991, p. 61).
} 
fortement les bouleversements engendrés par cette année du « tournant $»^{2}$, correspondant à une véritable Zeitenwende, tournant d'une époque mais aussi, au sens propre, tournant temporel. Christa Wolf note dans son journal que cette année 1989/1990 correspond à une période où les catégories, y compris celle du temps, se renversent et se retournent ${ }^{3}$, alors que Thomas Rosenlöcher souligne les phénomènes d'accélération à laquelle la société est soudainement soumise : "Le temps, autrefois à l'arrêt, est passé au galop, comme s'il tentait de rattraper les 40 années perdues ${ }^{4}$.

Ces trois témoignages datant de la période immédiatement postérieure à la chute du Mur confirment une différence dans la perception du temps que Heiner Müller avait déjà enregistrée au début des années 1980 lorsque, franchissant la frontière, il constate une "différence de civilisations, d'époques, de temps », voire un véritable « mur de temps $»^{5}$. Ailleurs, Müller caractérise cette différence comme un choc de « fuseaux horaires différents ", avec, à l'Ouest, la prédominance du "principe d'accélération » et à l'Est, celui du " ralentissement $»^{6}$.

On peut retenir que le temps est une « dimension fondamentale [...] dans laquelle l'être humain se situe à la fois comme être biologique et physique ainsi que comme être spirituel $»^{7}$. D'une part, le temps physique peut être objectivement mesuré, d'autre part, le temps peut être perçu différemment par les individus. Lorsqu'avec la chute du Mur de Berlin s'effondre également un "mur de temps", les horloges continuent à indiquer la même heure à l'Est comme à l'Ouest, mais la perception du temps change pour les Allemands de l'Est, soudainement confrontés à un ordre temporel différent.

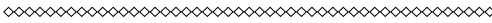

2. - Le terme Wende est à manier avec beaucoup de réserves puisqu'il a été d'abord utilisé par Egon Krenz comme signal pour un changement de cap au sein du SED.

3. - « Als sei ihm [dem Wendejahr] eine Achse eingezogen, um die herum die Zeit sich ,wendet'. Nun liegt unten, was vorher ,oben', also sichtbar war, und das - uns - bisher Unsichtbare liegt obenauf. » (27/9/1990). Christa Wolf, Ein Tag im Jahr. 1960-2000, München, btb, 2005, p. 459.

4. - «Die einstmals stillstehende Zeit ist in einen Galopp übergegangen, als wollte sie die verlorenen 40 Jahre wieder einholen. » Thomas Rosenlöcher, Die verkauften Pflastersteine. Dresdner Tagebuch, Frankfurt a. M., Suhrkamp, 1990, p. 44. Sauf indication contraire, nous traduisons.

5. - « einen Unterschied von Zivilisationen, von Epochen, von Zeit »; " Man fährt da wirklich durch eine Zeitmauer. » Heiner Müller, « Ich glaube an Konflikt. Sonst glaube ich an nichts. Ein Gespräch mit Sylvère Lotringer » [1981], in : Id., Werke 10. Gespräche 1 (1965-1987), hrsg. v. Frank Hörnigk, Frankfurt a. M., Suhrkamp, 2008, p. 175-223, ici p. 175.

6. - Heiner Müller, «Stirb schneller, Europa » (Gespräch mit Frank M. Raddatz) [1989], in : Id., Werke 11. Gespräche 2 (1987-1991), hrsg. v. Frank Hörnigk, Frankfurt a. M., Suhrkamp, 2008, p. 398-415, ici p. 414.

7. - « eine grundlegende Dimension [...], in der sich der Mensch sowohl als biologisch-physikalisches wie auch als geistiges Wesen verortet ». Norman Sieroka, Philosophie der Zeit. Grundlagen und Perspektiven, München, C.H. Beck, 2018, p. 10. 


\section{L'année 1989 comme « rupture temporelle »}

Cette rupture du temps vécue par les Allemands de l'Est a été à la fois exprimée par diverses métaphores et expliquée en termes sociologiques. Selon l'essayiste Lothar Baier, le Mur avait non seulement coupé une population du monde extérieur, mais aussi protégé une zone où le temps était disponible en abondance. L'irruption du temps occidental aurait donné " une nouvelle mesure à la vie des Allemands en RDA ", changement que Baier décrit avec une image empruntée à la physique : la « compensation de pression» (Druckausgleich) ${ }^{8}$. Selon lui, « le temps en expansion de l'Ouest déferle avec un sifflement inaudible à travers les valves soudainement ouvertes dans les espaces jusqu'alors fermés de l'Est, comme dans une chambre à vide devenue perméable. " " L'argumentation de Baier indique déjà que la conception du temps dépend de son inscription dans un système social particulier et que la perception du temps change lors de la transition vers une nouvelle forme de société. En ce sens, le temps fonctionne comme un «schéma cognitif », comme un "modèle préformateur de la perception ", pouvant conduire à une concurrence entre « différentes constructions collectives du temps » ainsi qu'entre «temps collectif» et « conscience individuelle du temps $»^{10}$. Comme le rappelle Aleida Assmann, le temps est une « construction culturelle " conduisant à la création d'horizons de sens qui entourent chaque individu depuis toujours ${ }^{11}$.

Quels sont donc ces différents " horizons de sens » temporels, liés aux sociétés est- et ouest-allemandes, qui se rencontrent en 1989 ? Heiner Müller avait évoqué les deux « fuseaux horaires » de l'accélération et du ralentissement, bien que le principe oriental de la décélération soit davantage le produit des réalités sociales que du modèle temporel revendiqué par l'État. En effet, le régime temporel de la RDA repose bien sur une " variante socialiste » du paradigme de la modernisation ${ }^{12}$ et le concept du progrès, certes dominé par l’idéologie. Ainsi, le régime temporel

8. - Lothar Baier, Volk ohne Zeit. Essay über das eilige Vaterland, Berlin, Wagenbach, 1990, p. 107, 103.

9. - « Die expandierende Zeit des Westens strömt mit unhörbarem Zischen durch die plötzlich geöffneten Ventile in die bisher geschlossenen Räume des Ostens wie in eine undicht gewordene Unterdruckkammer. » Ibid., p. 25.

10. - Stephanie Wodianka, «Zeit - Literatur - Gedächtnis », in : Astrid Erll et al. (dir.), Gedächtniskonzepte der Literaturwissenschaft: Theoretische Grundlegung und Anwendungsperspektiven, Berlin, de Gruyter, 2005, p. 179-201, ici p. 179sq.

11. - Aleida Assmann, Zeit und Tradition. Kulturelle Strategien der Dauer, Köln, Böhlau, 1999, p. $1,4$.

12. - Aleida Assmann, Ist die Zeit aus den Fugen? Aufstieg und Fall des Zeitregimes der Moderne, München, Carl Hanser, 2013, p. 82. 
de la modernité prévaut dans la mesure où, dans la société socialiste, « le concept physique de la 'flèche du temps' linéaire et irréversible est devenu la base irrévocable $»$ de l'ordre tempore ${ }^{13}$. La dynamique du " changement et de la transformation » ainsi que la démarcation par rapport à "l'ancien $~^{14}$ - ici avec la variante idéologique de 'l'homme nouveau' - font également partie des principes fondamentaux de la modernité. La principale différence tient aux perspectives ouvertes par l'avenir : alors que les sociétés hautement différenciées de la modernité tardive relient la conscience d'un temps linéaire à l'idée d'un avenir ouvert, sans que le cours de l'histoire s'oriente vers un but spécifique, les sociétés socialistes reposent sur l'idée marxiste d'une histoire téléologique avec, comme corrélat, un avenir fermé ${ }^{15}$. Ainsi, la conception idéologique du progrès en RDA considérait le socialisme comme promesse d'un salut, il représentait « l'espoir de posséder l'avenir $»^{16}$.

Cette orientation téléologique vers un avenir supposé meilleur a façonné des générations, mais la mise en œuvre concrète de ces idéaux dans le cadre du socialisme réellement existant $\mathrm{a}$, au contraire, conduit à la propagation progressive d'un sentiment d' ' anarchie temporelle ${ }^{17}$ et de stagnation, au lieu d'un mouvement et d'une progression constante. Bien qu'omniprésents dans le discours public, des concepts tels que " histoire ", « développement " ou " progrès ", décrits par Reinhart Koselleck comme des " concepts de mouvement ${ }^{18}$ en raison de leur potentiel sémantique de mouvement et d'accélération, ont perdu tout fondement en RDA, conduisant à ce que les prémisses idéologiques et la perception subjective du temps divergent de plus en plus. Une étude sociologique a d'ailleurs pointé un certain nombre de facteurs temporels propres à la société est-allemande conduisant à ce que le rythme de vie soit effectivement ressenti comme plus lent et que le temps semble être une ressource largement disponible ${ }^{19}$.

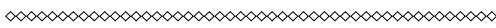

13. - Ibid., p. 24.

14. - Ibid., p. 23sq.

15. - Cf. à ce sujet Hartmut Rosa, Beschleunigung. Die Veränderung der Zeitstrukturen in der Moderne, Frankfurt a. M., Suhrkamp, 2005, p. 27.

16. - Martin Sabrow, "Sozialismus », in : Id. (dir.), Erinnerungsorte der DDR, München, C. H. Beck, 2009, p. 188-204, ici p. 188.

17. - Sandrine Kott, Le communisme au quotidien. Les entreprises d'État dans la société est-allemande, Paris, Belin, 2001, p. 296.

18. - Reinhart Koselleck, " "Neuzeit”. Zur Semantik moderner Bewegungsbegriffe », in : Id., Vergangene Zukunft. Zur Semantik geschichtlicher Zeiten [1979], Frankfurt a. M., Suhrkamp, 1989, p. 300-348, notamment p. 339-348.

19. - Parmi ces facteurs, on trouve un avenir hautement structuré qui permet de vivre au présent, la régularité du quotidien, la culture de l'attente ou encore la limitation des options pour agir. Cf. Wilhelm Hofmann, «Zusammenprall der Zeitkulturen: Lebenstempo und Zeitempfinden 
Le nouveau régime temporel auquel les Allemands de l'Est ont été confrontés après la chute du Mur se caractérise avant tout par deux aspects : accélération et manque d'orientation concernant l'avenir. Selon le sociologue Hartmut Rosa, l'année 1989 marque définitivement le passage de la modernité à la modernité tardive, la coïncidence des révolutions politique et numérique faisant que la perception fondamentale du temps était désormais celle d'une accélération accrue dans tous les domaines de la sociétée ${ }^{20}$. En même temps, on perçoit une tendance à la «paralysie sociale » dans les sociétés occidentales, résultant d'un manque d'options pour l'avenir ${ }^{21}$. Dès 1989 , la sociologue Helga Nowotny avait d'ailleurs expliqué à quel point l'horizon ouvert de l'avenir, synonyme de progrès et d'amélioration des conditions de vie, se dissolvait ou restait « plat et immobile » dans les sociétés occidentales ${ }^{22}$. «Les attentes à l'égard de l'avenir sont devenues modestes ", écrit également Aleida Assmann, l'avenir s'étant transformé « d'un objet d'attente et d'espoir en un objet d'inquiétude. ${ }^{23}$ Assmann souligne également que le régime temporel de la modernité, orienté vers l'avenir, est remplacé par un ordre temporel de plus en plus centré sur le passé lequel se voit accorder une place toujours plus grande dans le présent. Ce constat avait également été fait par Hans Ulrich Gumbrecht, selon lequel « les passés [inondent] notre présent, la perfection de la mémoire électronique jouant un rôle central $»^{24}$.

Cette évolution du régime temporel de la modernité vers la modernité tardive, brièvement esquissée ici, a aussi bien affecté les sociétés occidentales qu'orientales. Mais comme ces dernières se trouvaient en plein bouleversement sociétal, l'expérience temporelle particulière de la modernité tardive, celle de l'accélération dans tous les domaines de la vie, a été perçue avec encore plus de puissance. En effet, les Allemands de l'Est ont été bien plus nombreux que leurs voisins ouest-allemands à répondre que «le temps va à toute vitesse » lors d'un sondage en $1995^{25}$. Leur rythme de vie s'est considérablement accéléré après 1989.

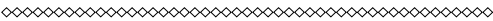

in Ostdeutschland vor und nach der Wiedervereinigung ", in : Hartmut Rosa (dir.), fast forward Essays zu Zeit und Beschleunigung. Standpunkte junger Forschung, Hamburg, Ed. Körber-Stiftung, 2004, p. 57-72.

20. - Hartmut Rosa, Beschleunigung, op. cit., p. 40, 335.

21. - Ibid., p. 41.

22. - Helga Nowotny, Eigenzeit. Entstehung und Strukturierung eines Zeitgefühls, Frankfurt a. M., Suhrkamp, 1989, p. 51.

23. - Aleida Assmann, Ist die Zeit aus den Fugen?..., op. cit., p. 12sq.

24. - Hans Ulrich Gumbrecht, Unsere breite Gegenwart, traduit de l'anglais par Frank Born, Berlin, Suhrkamp, 2010, p. 16.

25. - Wilhelm Hofmann, «Zusammenprall der Zeitkulturen... », op. cit., p. 69. 
La rupture temporelle vécue par les Allemands de l'Est en 1989 pourrait se résumer comme suit : à l'immobilisme social succède une énorme poussée d'accélération qui ne débouche toutefois pas sur un avenir ouvert, pouvant être façonné et exploité individuellement, mais sur un avenir bloqué. En outre, on assiste à une revalorisation du passé qui va toutefois de pair avec une remise en question de l'histoire spécifique de la RDA, largement associée à des connotations négatives.

\section{Représentations littéraires et artistiques}

Comme les exemples cités au début le montrent, les écrivains se sont immédiatement saisis de ce phénomène et la littérature est l'un des lieux où cette rupture temporelle, témoignant également d'un décalage entre temps individuel et temps historique, a trouvé son expression. C'est le cas pour les textes qui ont immédiatement paru après la chute du Mur, mais c'est une thématique qui persiste jusque dans des œuvres très récentes ${ }^{26}$, confirmant qu'il s'agit bien d'une problématique ressentie comme existentielle.

Le présent numéro de Germanica ${ }^{27}$ souhaite interroger la façon dont la littérature en tant que mise en fiction d'expériences personnelles et culturelles et forme particulière d'une "introspection des sociétés $»^{28}$ traduit et figure ce changement de la perception du temps lié au passage d'une époque à l'autre, d'une société à l'autre. Ce faisant, il est bien évident que ni la littérature ni les autres formes artistiques comme le cinéma et le théâtre dont il sera également question ne sont à considérer comme une simple illustration des modèles sociologiques évoqués plus haut. Les articles réunis ici interrogent de différentes façons un certain nombre d'éléments. Dans quelle mesure la littérature ou le cinéma rendent-ils compte de la disparition d'une conception du temps linéaire, centrée sur l'idée d'un avenir utopique et fermé, propre à la RDA, et de l'apparition d'un temps accéléré sans ancrage possible dans un avenir incertain ? Les œuvres mettent-elles en scène une réflexion sur le passé pour pallier le ressenti d'une discontinuité de l'expérience subjective depuis 1989 ? Comment la littérature exprime-t-elle la quête d'un temps individuel se distanciant à la fois de l'ancien et du nouveau régime temporel ? La perception du temps

26. - Par exemple dans le dernier roman de Lutz Seiler, Stern 111, paru en 2020.

27. - Ce numéro s'appuie sur certaines communications présentées lors de l'atelier « Zeiterfahrung und gesellschaftlicher Umbruch in Fiktionen der Post-DDR-Literatur » que j'ai organisé à l'occasion du $26^{\text {ème }}$ Deutscher Germanistentag (22-25/09/2019, Universität des Saarlandes, Sarrebruck), consacré à la thématique du « temps » («Zeit »).

28. - Hartmut Böhme, «Zur Gegenstandsfrage der Germanistik und Kulturwissenschaft », Jahrbuch der Deutschen Schillergesellschaft, t. XLII (1998), p. 476-485, ici p. 480. 
peut s'exprimer par des réflexions des narrateurs ou des personnages qui thématisent directement un rapport modifié au temps, mais elle peut également prendre des formes plus discrètes dans des textes qui, a priori, ne racontent ou ne représentent pas l'événement et ses conséquences ou ne le font que peu. Ainsi, l'expérience d'un changement de temporalité peut être figurée par des moyens esthétiques comme des symboles, des métaphores, des constructions narratives particulières ou des intertextes.

Les contributions englobent une période assez vaste, couvrant des productions parues sur presque trente ans, allant de l'analyse d'un des premiers Wenderomane, "romans du tournant $»^{29}$, aujourd'hui peu connu, jusqu'au long-métrage Gundermann (2018) d'Andreas Dresen. Alors que les deux premières contributions se concentrent sur des textes mettant en avant des ruptures biographiques directement liées aux bouleversements de 1989, les articles suivants s'intéressent à des œuvres qui embrassent des perspectives plus larges, avec un questionnement plus prononcé sur le temps historique.

Ce volume s'ouvre avec une contribution de Janine Ludwig consacrée au roman Schlehweins Giraffe (1992) de Bernd Schirmer, l'un des premiers romans parus après 1989 à dévoiler, avec ironie et humour, les ravages sociaux de l'unification dans un petit groupe d'amis, intellectuels et chercheurs ayant tous perdu leur emploi. L'analyse en termes de temporalité montre que ce roman témoigne en effet de cette accélération rapide dans tous les domaines de la vie décrite plus haut, mais qu'il renvoie également à un autre phénomène analysé par Paul Virilio : 1 '« inertie polaire », traduit en allemand par « der rasende Stillstand ». Le narrateur, tombé hors du temps par la perte de ses repères, semble désormais vivre à l'arrêt. Rien de significatif ne se passe au présent, il est pris en étau entre un passé dont on l'invite à se distancier et un avenir vers lequel il ne peut se projeter. En se référant aux catégories sociologiques du « temps quotidien », du « temps biographique » et du "temps historique ", l'article montre comment les bouleversements de 1989/9o créent une tension entre ces trois niveaux, alors qu'un « temps sacré » qui ferait sens fait défaut.

Le premier récit de Jenny Erpenbeck, Geschichte vom alten Kind, publié en 1999, interroge à sa manière la rupture temporelle de 1989. Appartenant à une tout autre génération que Bernd Schirmer, la réflexion

29. - Ce n'est pas le lieu ici de retracer les débats autour des différentes dénominations et définitions de la littérature du " tournant » et de « l'après-tournant », nous renvoyons à la synthèse proposée par Sonja Kersten : « Mauerfall- , Post-DDR-, Vereinigungs-, Nachwende- oder doch Wendeliteratur? Eine kleine Expedition durch einen großen Begriffsdschungel », literaturkritik.de $\mathrm{n}^{\circ}$ 10, octobre 2015, Schwerpunkt 25 Jahre Deutsche Einheit, https://literaturkritik.de/id/21125, consulté le 10/05/2021. 
sur les événements de 1989 n'est pas moins importante chez Erpenbeck qui, toutefois, a recours à un traitement radicalement différent. Carola Hähnel-Mesnard montre dans quelle mesure le thème de la métamorphose est un dispositif littéraire susceptible de saisir la relation entre expérience historique et perception individuelle du temps. La transformation physique d'une jeune femme en enfant peut ainsi être lue comme une parabole des bouleversements de 1989 et interroger le conflit entre temps individuel et temps historique, l'analyse narratologique permettant de mettre en évidence cette tension. Le renoncement à une contextualisation précise fait du récit un exemple paradigmatique d'une représentation non référentielle des bouleversements de 1989.

Avec son analyse du roman Abschied von den Feinden (1995) de Reinhard Jirgl, mettant en scène le destin de deux "frères ennemis " avant et après la chute du Mur, Johanna Vollmeyer s'intéresse à un auteur à l'écriture particulière qui s'est imposé depuis le début des années 1990 comme observateur critique aussi bien du passé que du présent. Avec Jirgl, on dépasse le niveau de la perception d'une rupture temporelle intervenue en 1989, car son roman - comme son œuvre en général interroge de manière plus globale les différents régimes temporels dans l'Allemagne divisée, puis unifiée, en montrant que la chronopolitique est un mécanisme de domination propre à tout régime politique. L'article interroge plus particulièrement le thème du train auquel sont associées différentes conceptions du temps, permettant un voyage dans le temps tout en symbolisant les ruptures dans les biographies des personnages. L'observation critique des différentes couches du passé et du présent repose sur une remise en question de la notion du temps comme unité homogène, linéaire et vide, privilégiant une approche subjective où domine l'imbrication des différents moments temporels, se matérialisant de manière performative.

Fanny Perrier s'intéresse quant à elle au roman Willenbrock de Christoph Hein, paru en 2000, au centre duquel se trouve le personnage éponyme qui représente de prime abord la réussite professionnelle et économique d'un Allemand de l'Est après la chute du Mur, se trouvant ainsi en phase avec cette nouvelle époque d'un temps accéléré. Toutefois, lorsque le personnage est confronté à son passé, la progression linéaire et apparemment sans rupture de sa vie se trouve bousculée. En analysant le roman de Hein à travers le prisme de la philosophie de l'histoire de Walter Benjamin, qui avait fortement influencé l'auteur est-allemand, Fanny Perrier montre à quel point le roman insiste sur l'importance de la reconnaissance du passé dans le présent. Chez Hein, on observe donc 
moins les conséquences d'une unification hâtive que celles d'un passé refoulé sur lequel on a voulu tirer un trait. Si Willenbrock cherche à la fin une sorte de croyance transcendante, cela pourrait précisément renvoyer à l'absence - et la recherche - d'un " temps sacré » permettant de faire sens, abordé dans l'article de Janine Ludwig.

La contribution de Matthias Kandziora élargit encore la perspective pour s'intéresser aux implications temporelles d'un monde globalisé dans l'œuvre de Christa Wolf. Si le dernier roman que l'auteure a publié de son vivant, Stadt der Engel oder The Overcoat of Dr. Freud (2010), est à ce tire exemplaire, l'article s'intéresse d'abord aux signes précurseurs de la mondialisation tels que Wolf les détecte dans la catastrophe de Tchernobyl, au centre de son récit Störfall (1987). Ensuite, il réexamine le roman de 2010 et le rapport modifié au temps qu'exprime la narratrice, entre réflexions sur le passé et perception du « tournant » de 1989, l'expérience d'un séjour aux États-Unis au début des années 1990 et le moment de la narration au milieu des années 2000. La narratrice réinterroge sa relation au temps à travers la rencontre avec le peuple indigène des Hopis qui lui sert à la fois de référence lorsqu'elle évoque les bouleversements des années 1989/9o, et de prisme pour interroger les conséquences d'un monde soumis à l'accélération et à la globalisation.

Valérie Carré s'intéresse ensuite au long-métrage Gundermann d'Andreas Dresen et de Laila Stieler, sorti sur les écrans en 2018. Ce biopic sur le chanteur est-allemand Gerhard Gundermann qui travaillait à temps complet comme conducteur d'une excavatrice dans une mine à ciel ouvert tout en étant collaborateur de la Stasi, est construit sur différentes strates temporelles dont l'article analyse agencement et signification. Il relève également les procédés et symboles qui mettent en évidence la concurrence entre temps linéaire et temps cyclique, ce dernier renvoyant à un temps mythique, celui de la régénération et du renouveau permettant, entre autres, de transcender le destin individuel mis en scène dans le film et de construire un récit transmettant de façon intense l'expérience collective de l'époque.

Les contributions scientifiques de ce dossier se terminent avec un article qui considère les conséquences d'une expérience sociétale et temporelle modifiée non pas à partir d'une œuvre, mais à partir d'une pratique artistique, à savoir celle des collectifs de théâtre. En comparant deux périodes très différentes, les années 1960/1970 et le début du XXI ${ }^{\mathrm{e}}$ siècle, Vera Nitsche interroge les répercussions de «l'esprit du temps » respectif sur les pratiques artistiques et leur organisation. Ainsi, les collectifs de théâtre étaient autrefois considérés comme précurseurs d'un modèle 
socialiste - ce qui, rétrospectivement, confère aux discours qu'ils portent une note étrangement anachronique. En revanche, après la chute du "socialisme réellement existant " et les bouleversements politiques et socio-économiques qui ont suivi, on va jusqu'à considérer les nouveaux collectifs comme expression de la dérégulation néolibérale. L'article interroge ce paradoxe tout en analysant les choix esthétiques faits à différentes époques.

Nous avons également voulu dans ce dossier donner la parole à une auteure dont l'œuvre porte les traces de cette rupture temporelle de 1989, que ce soit d'un point de vue thématique ou esthétique. En effet, Julia Schoch fait partie des auteures de la littérature « post-RDA » qui interrogent de façon insistante les conséquences de cette expérience historique particulière, de cette « rupture absolue » ouvrant sur un monde où, selon elle, plus rien n'allait de $\operatorname{soi}^{30}$. Dans son roman Mit der Geschwindigkeit des Sommers, paru en 2009, l'auteure construit un personnage qui n'a plus d'ancrage dans le temps : le présent dans lequel elle vit désormais ne fait pas sens, les promesses d'avenir du nouveau monde conduisent à un « galop sur place», alors que les tentatives nostalgiques de se réfugier dans le passé, d'y retrouver le fil des visions antérieures d'un avenir assuré, échouent. Nous publions pour la première fois des extraits de ce roman dans la traduction inédite de Leïla Pellissier : À la vitesse de l'été. Par ailleurs, Julia Schoch a bien voulu répondre à un certain nombre de questions en lien avec la thématique du temps dans son œuvre, ses réponses pour lesquelles nous lui remercions tout particulièrement, parachèvent ce dossier.

30. - « Es ist die Erfahrung eines absoluten Bruchs - und die Wende war so ein Bruch. Es ist die grundlegende Erfahrung, dass das, was da ist, nicht selbstverständlich ist. » Susanne Leinemann/ Antje Schmelcher, "Generation Trabant. Angekommen im neuen Deutschland? Zonenkinder im Gespräch », Die Welt, 9/11/2002. Pour une analyse de la perception du temps dans l'œuvre de Julia Schoch, cf. Carola Hähnel-Mesnard, "Geschichte und Zeiterfahrung in der Prosa von Julia Schoch », in : Christiane Caemmerer, Walter Delabar, Helga Meise (dir.), Fräuleinwunder. Zum literarischen Nachleben eines Labels, Frankfurt a. M., Peter Lang, 2017, p. 31-49. 